\title{
Selectfluor 作用下二芳基二硫醚和醇的双亚磺酰化反应制备亚磺酸酯
}

\author{
刘爱遥 ${ }^{a}$ 刘 江 ${ }^{a} \quad$ 梅海波*,a,b \\ Gerd-Volker Röschenthaler ${ }^{c}$ 韩建林*,a \\ ( ${ }^{a}$ 南京林业大学化学工程学院 江苏省林业资源高效加工利用协调创新中心＼cjkstart南京 210037) \\ ( ${ }^{b}$ 青岛科技大学化学与分子工程学院 山东省生化分析重点实验室 青岛 266042) \\ ( ${ }^{c}$ 不来梅雅各布大学生命科学与化学学院 不来梅 28759)
}

\begin{abstract}
摘要 报道了一种新型的由二芳基二硫醚和醇双亚磺酰化反应来制备亚磺酸酯的方法. 在 Selectfluor 作为氧化剂的条 件下，一分子二硫醚与两分子醇反应，能够顺利转化得到两分子亚磺酸酯产物。该反应无需金属催化剂，在简单温和 的条件下即可进行, 反应产率很高. 反应底物适用性广, 含有不同取代基的二硫醚和醇, 包括天然的手性醇类化合物, 均能很好地发生反应得到相应的产物. 该方法实现了简单条件下通过醇的亚磺酰化反应高效地构建亚磺酸酯, 为亚磺 酸酯及其衍生物的合成提供了一条新的途径.
\end{abstract}

关键词 Selectfluor; 二硫醚; 亚磺酰化; 亚磺酸酯; C一O 键断裂

\section{Selectfluor-Promoted Twofold Sulfination of Alcohols for the Synthesis of Sulfinic Ester from Diaryldisulfides}

\author{
Liu, Aiyao $^{a} \quad$ Liu, Jiang $^{a} \quad$ Mei, Haibo ${ }^{*, a, b}$ Röschenthaler, Gerd-Volker ${ }^{c} \quad$ Han, Jianlin ${ }^{*, a}$ \\ ( ${ }^{a}$ Jiangsu Co-Innovation Center of Efficient Processing and Utilization of Forest Resources, \\ College of Chemical Engineering, Nanjing Forestry University, Nanjing 210037, China) \\ $\left({ }^{b}\right.$ Shandong Key Laboratory of Biochemical Analysis, College of Chemistry and Molecular Engineering, \\ Qingdao University of Science and Technology, Qingdao 266042, China) \\ ( ${ }^{c}$ Department of Life Sciences and Chemistry, Jacobs University Bremen gGmbH, Bremen 28759, Germany)
}

\begin{abstract}
An oxidative twofold sulfination of alcohol with Selectfluor as an efficient oxidant was developed. This reaction proceeded smoothly achieving the unprecedented sulfination transformation of varieties of diaryldisulfides and alcohols under simple conditions, affording the corresponding sulfinic esters in excellent yields. The current reaction provides a new and convenient strategy for the preparation of sulfinic esters.

Keywords Selectfluor; disulfide; sufination; sulfinic ester; $\mathrm{C}-\mathrm{O}$ bond cleavage
\end{abstract}

\section{Introduction}

Sulfinic esters and their derivatives belong to highly important organic molecules because they are versatile synthetic intermediates for the preparation of sulfonyl group-containing compounds in organic chemistry ${ }^{[1]}$ Also, these structural units widely exist in many pharmaceuticals and biologically active molecules. ${ }^{[2]}$ Thus, development of the corresponding synthetic methods attracts growing research interests in recent years. The traditional methods for the synthesis of sulfinic esters were esterification of sulfinic acids in the presence of catalyst, additives or condensation reagents. ${ }^{[3]}$ Alternatively, sulfinic esters could be prepared from thiosulfinic $S$-esters, ${ }^{[4]}$ sulfonamides ${ }^{[5]}$ and sodium sulfinates. ${ }^{[6]}$ However, these starting materials are not stable and the related reactions usually need harsh conditions. Oxidation of more stable and readily available disulfides or thiols represented another strategy for the preparation of sulfinic esters with the aid of oxidants, such as chlorine, $N$-bromosuccinimide (NBS), meta-chloroperoxy-

\footnotetext{
* Corresponding authors. E-mail: meihb@njfu.edu.cn; hanjl@njfu.edu.cn Received March 3, 2020; revised May 6, 2020; published online May 15, 2020.

Project supported by the National Natural Science Foundation of China (No. 21761132021), the German Research Foundation (No. 362/74-1) and the Open Project of Chemistry Department of Qingdao University of Science and Technology (No. QUSTHX202005).

国家自然科学基金(No. 21761132021)、德国研究基金(No. 362/74-1)和青岛科技大学化学学部开放课题(No. QUSTHX202005)资助项目.
} 
benzoic acid ( $m$-CPBA), transition-metal/oxygen and even additives. $^{[7]}$ Our group has developed two examples on $\mathrm{Cu}$-catalyzed aerobic coupling reactions with sulfonyl hydrazides $^{[8 \mathrm{a}]}$ and $\beta$-keto sulfones ${ }^{[\mathrm{bb}]}$ as the coupling partners respectively. Very recently, the $\mathrm{Li}^{[9 \mathrm{a}]}$ and $\mathrm{Lei}^{\left[{ }^{[9 b]}\right.}$ groups independently developed an oxidative reaction of odiferous thiols for the synthesis of sulfinic esters under electrochemical conditions. However, these reactions usually suffered from several limitations including the use of transition-metal catalysts, narrow alcohol scope, poor functional group tolerance and moderate yields. ${ }^{[10]}$

Selectfluor is a highly reactive compound, and has been extensively employed as an efficient fluorinating reagent. ${ }^{[11-12]}$ Furthermore, it could be used as a mild oxidant in the $\mathrm{C}-\mathrm{H}$ bond functionalization reaction via fluorine radical intermediate. ${ }^{[13]}$ However, Selectfluor-promoted oxidative transformations of sulfur-containing compounds, such as thiols, sulfides and disulfides still remain great challenges. Selectfluor was reported for the selective thiolation of indoles with thiols, affording 3-sulfenyl indoles as products (Scheme 1a). ${ }^{[14]}$ Oxidation of disulfides by Selectfluor in the presence of water resulted in thiosulfonates via the sequence of formation of sulfonium, substitution by water and deprotonation (Scheme 1b). ${ }^{[15]}$ Selectfluor also could convert thioglycosides into sulfoxide in the presence of base via a similar deprotonation process (Scheme 1, c). ${ }^{[16]}$ To the best of our knowledge, oxidation of disulfide or thiols by Selectfluor to give sulfinates has never been explored until now. Herein, a Selectfluor-promoted oxidative reaction of disulfides for the synthesis of sulfinic esters under simple conditions (Scheme 1d) is developed. It should be mentioned that the reaction was carried out under mild conditions without use of any transition-metals or additives. Furthermore, this transformation demonstrates a new oxidative reaction of Selectfluor for functionalization
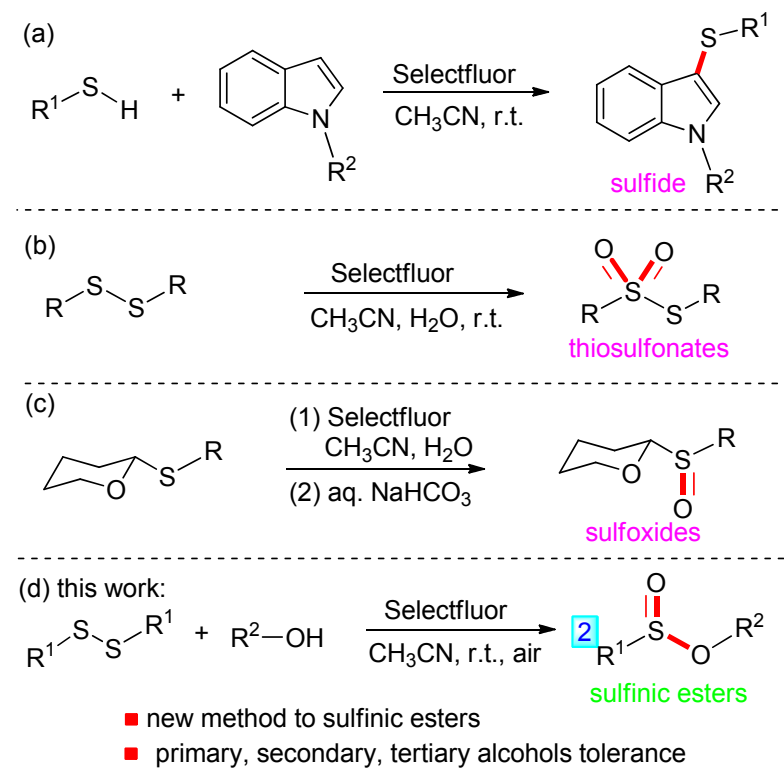

Scheme 1 Selectfluor-promoted transformations of disulfides, sulfides or thiols of disulfides, and also represents an efficient, convenient strategy for the synthesis of sulfinic esters.

\section{Results and discussion}

Our initial study was carried out with diphenyl disulfide (1a) and ethanol (2a) as model substrates in the presence of Selectfluor in acetonitrile at room temperature under a nitrogen atmosphere (Table 1). It was found that the reaction between 1a and $\mathbf{2 a}$ did happen with the use of 2.0 equiv. of Selectfluor at room temperature, resulting in the corresponding sulfinic ester $\mathbf{3 a}$ in $45 \%$ yield (Entry 1 ). In particular, both of the two phenylthio moieties are transferred into the desired sulfinic ester 3a. Interestingly, we found that the yield of this reaction obviously depends on the loading amount of Selectfluor (Entries 1 4). The results of Entries 1 4 showed that 4.0 equiv. of Selectfluor was the best one and the yield was increased to $82 \%$ (Entry 3 ). Intrigued by these results, we continued the optimization by screening a series of different solvents. Switching acetonitrile to other solvents, no improvement was found on the reaction outcome (Entries 5 11). In particular, no desired product 3a was detected at all in the cases of tetrahydrofuran (THF), dichloromethane, 1,2-di- chloroethane, dimethylsulfoxide (DMSO) and chlorobenezene. Since oxygen is needed for the transformation, we carried out the reaction under an air and oxygen atmosphere. We were pleased that the chemical yield was further increased to 94\% when the reaction was conducted under air (Entry 12). The variations on the reaction temperature were not

Table 1 Optimization of the reaction conditions ${ }^{a}$

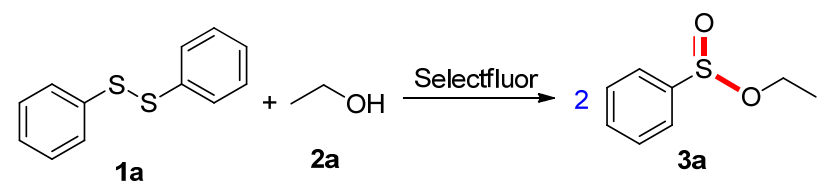

\begin{tabular}{cclccc}
\hline Entry & $\begin{array}{c}\text { Selectfluor/ } \\
\text { equiv. }\end{array}$ & Solvent & Atmosphere & \multicolumn{2}{c}{$T{ }^{\circ} \mathrm{C}$ Yield ${ }^{b} \%$} \\
\hline 1 & 2 & $\mathrm{CH}_{3} \mathrm{CN}$ & $\mathrm{N}_{2}$ & r.t. & 45 \\
2 & 3 & $\mathrm{CH}_{3} \mathrm{CN}$ & $\mathrm{N}_{2}$ & r.t. & 64 \\
3 & 4 & $\mathrm{CH}_{3} \mathrm{CN}$ & $\mathrm{N}_{2}$ & r.t. & 82 \\
4 & 5 & $\mathrm{CH}_{3} \mathrm{CN}$ & $\mathrm{N}_{2}$ & r.t. & 63 \\
5 & 4 & $\mathrm{THF}$ & $\mathrm{N}_{2}$ & r.t. & 0 \\
6 & 4 & $\mathrm{DMF}_{2}$ & $\mathrm{~N}_{2}$ & r.t. & 81 \\
7 & 4 & $\mathrm{CH}_{2} \mathrm{Cl}_{2}$ & $\mathrm{~N}_{2}$ & r.t. & 0 \\
8 & 4 & $\mathrm{ClCH}_{2} \mathrm{CH}_{2} \mathrm{Cl}$ & $\mathrm{N}_{2}$ & r.t. & 0 \\
9 & 4 & $\mathrm{CH}_{3} \mathrm{NO}_{2}$ & $\mathrm{~N}_{2}$ & r.t. & 79 \\
10 & 4 & $\mathrm{DMSO}$ & $\mathrm{N}_{2}$ & r.t. & 0 \\
11 & 4 & $\mathrm{PhCl}_{1}$ & $\mathrm{~N}_{2}$ & r.t. & 0 \\
12 & 4 & $\mathrm{CH}_{3} \mathrm{CN}$ & air & r.t. & 94 \\
13 & 4 & $\mathrm{CH}_{3} \mathrm{CN}$ & $\mathrm{O}_{2}$ & r.t. & 90 \\
14 & 4 & $\mathrm{CH}_{3} \mathrm{CN}$ & air & 0 & 40 \\
15 & 4 & $\mathrm{CH}_{3} \mathrm{CN}$ & air & 50 & 52 \\
16 & 0 & $\mathrm{CH}_{3} \mathrm{CN}$ & air & r.t. & 0 \\
\hline
\end{tabular}

${ }^{a}$ Reaction conditions: 1a $(0.2 \mathrm{mmol})$, ethanol 2a $(1 \mathrm{~mL})$, solvent $(4 \mathrm{~mL})$, at room temperature for $4 \mathrm{~h} .{ }^{b}$ Isolated yield based on $\mathbf{1 a}$. 
successful (Entries 14, 15). Finally, we carried out the reaction without the addition of Selectfluor in acetonitrile for $4 \mathrm{~h}$. No reaction occurred with all the staring material remaining (Entry 16). This result suggests Selectfluor is essential for this transformation.

The next goal was the examination of the substrate scope for this oxidative coupling reaction under the optimized conditions. First, the reactions of ethanol $\mathbf{2 a}$ with varieties of disulfides 1 was explored (Table 2). In general, excellent yields were obtained from most of the reactions with aryl disulfides (3a $\sim \mathbf{3 h}, 71 \% \sim 98 \%$ yields). Aryl disulfides bearing different substituents on the aromatic ring, even methoxyl (3c), fluoro (3d, 3e) and nitro (3h) groups, were all well tolerated, providing the desired product in good yields. The position of substituted group on the aromatic ring showed almost no effect on the yield (3d and $\mathbf{3 e}$ ). Importantly, the disulfides featuring heterocyclic units, such as pyridyl, thienyl and tetrazol could also be converted into the corresponding sulfinic esters $(\mathbf{3 i} \sim \mathbf{3 k})$ with $75 \%, 83 \%$, and $30 \%$ yield, respectively.

Then, we continued to explore the substrate scope of alcohols by reacting with diphenyl disulfide (1a) under the standard conditions (Table 2). Several linear aliphatic alcohols were examined in the current system, and all the reactions proceeded smoothly resulting in the expected products $3 \mathbf{3} \sim \mathbf{3 r}$ in excellent yields $(88 \% \sim 98 \%)$. The

Table 2 Substrate scope studies ${ }^{a}$

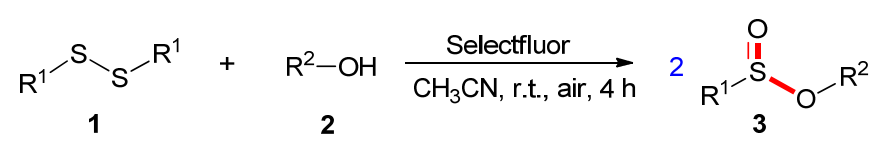<smiles>CCOS(=O)c1ccccc1</smiles>

3a, $94 \%$<smiles>CCOS(=O)c1cc(Cl)cc(Cl)c1</smiles>

3g, $98 \%$<smiles>CCOS(=O)c1ccc(C)cc1</smiles>

3b, $86 \%$<smiles>CCOS(=O)c1ccc(OC)cc1</smiles>

3c, $71 \%$<smiles>CCOS(=O)c1ccccc1F</smiles>

3d, $88 \%$<smiles>CCOS(=O)c1cccc(F)c1</smiles>

$3 \mathbf{e}, 89 \%$<smiles>CCOS(=O)c1ccc(Cl)cc1</smiles>

3f, $95 \%$<smiles>CCOS(=O)c1cccc([N+](=O)[O-])c1</smiles>

3h, $91 \%$<smiles>CCOS(=O)c1ccccn1</smiles>

$3 \mathbf{i}, 75 \%$<smiles>CCOS(=O)c1cccs1</smiles>

3j, $83 \%$<smiles>CCOS(=O)c1nnnn1-c1ccccc1</smiles><smiles>COS(=O)c1ccccc1</smiles>

$31,94 \%$<smiles>CCCOS(=O)c1ccccc1</smiles>

$3 \mathrm{~m}, 96 \%$<smiles>CCCCOS(=O)c1ccccc1</smiles>

3n, $98 \%$<smiles>CCCCCOS(=O)c1ccccc1</smiles>

3o, $95 \%$<smiles>CC(C)OS(=O)c1ccccc1</smiles>

3p, $98 \%$<smiles>CC(C)COS(=O)c1ccccc1</smiles>

3q, $88 \%$<smiles>CC(C)CCOS(=O)c1ccccc1</smiles>

$3 \mathbf{r}, 91 \%$<smiles>O=S(OC1CCCCCCCCCCC1)c1ccccc1</smiles>

$3 \mathbf{x}, 70 \%{ }^{b}$<smiles>CC(C)(C)OS(=O)c1ccccc1</smiles>

3s, $57 \%$<smiles>CC(C)(C)[13CH3]</smiles><smiles>O=S(OC1CCCC1)c1ccccc1</smiles>

3u, $80 \%$<smiles>O=S(OC1CCCCC1)c1ccccc1</smiles>

3v, $84 \%$<smiles>O=S(OC1CCCCCC1)c1ccccc1</smiles>

$3 t, 98 \%$<smiles>CCOC(=O)COS(=O)c1ccccc1</smiles><smiles>Cc1ccc(S(=O)OCc2ccccn2)cc1</smiles>

c) natural chiral alcohol scope<smiles>CC(C)C1CC[C@@H](C)C[C@H]1OS(=O)c1ccccc1</smiles>

3ad, $74 \%,{ }^{b} 1: 1 d r^{c}$<smiles>CC1(C)[C@H]2CC[C@@]1(C)[C@H](OS(=O)c1ccccc1)C2</smiles>

3ae, $56 \%,{ }^{b} 1: 1 d r^{c}$

${ }^{a}$ Reaction conditions: $1(0.2 \mathrm{mmol}), \mathbf{2}(1 \mathrm{~mL})$, Selectfluor (4 equiv.), $\mathrm{CH}_{3} \mathrm{CN}(4 \mathrm{~mL})$, at room temperature under air for $4 \mathrm{~h}$; isolated yield. ${ }^{b} 5.0$ equiv. of alcohol was used. ${ }^{c} d r$ was determined by ${ }^{1} \mathrm{H}$ NMR. 
length of the chain has no effect on the chemical yield, and even in the case of pentanol (3o), 95\% yield was obtained. Besides primary alcohols, secondary and tertiary alcohols were also suitable substrates for this system. For example, bulky isopropanol could react with diphenyl disulfide (1a) very well resulting in the product $\mathbf{3 p}$ with $98 \%$ yield. Notably, even more bulky tert-butanol also could be converted into the corresponding sulfinic ester 3s with $57 \%$ yield. These results underscore the great advantages to the previous reports that tertiary alcohols can not be tolerated. ${ }^{[8-9]}$ It is very interesting that the alcohol substrate also could be extended to cyclic alcohols. These results indicated that cyclic alcohols containing four- (3t), five- (3u), six- (3v), and seven-membered (3w) aliphatic rings all worked very well in this oxidative system resulting in the corresponding sulfinic esters in good to excellent chemical yields $(80 \%$ $98 \%$ ). Of particular interest was that even the macrocyclic compound, cyclododecanol, was well tolerated and the corresponding sulfinic ester $\mathbf{3 x}$ was obtained in $70 \%$ yield. To further demonstrate the utility of this oxidative sulfination reaction, several alcohols bearing functional groups were tried in this system. For example, 1-(hydroxymethyl)adamantane could react with disulfide 1a very well in the presence of Selectfluor, providing the desired product $\mathbf{3 y}$ in $86 \%$ yield. Also, the alcohol containing ester group or pyridyl group was also suitable for this reaction, affording the products $\mathbf{3 z}$ and $3 \mathbf{a b}$ in $46 \%$ and $82 \%$ yield respectively. Phenol was examined as a substrate for this reaction, however, the reaction of phenol with 1a did not occur at all. Finally, this strategy was applied in the reaction of chiral natural alcohol such as $(-)$-menthol and $(-)$-borneol (Table 2). The reaction also proceeded very well to provide the expected sulfinic esters in good yields (3ad and 3ae) and poor diastereoselectivities $(d r=1: 1)$. It should be mentioned that the two diastereomers could not be separated by the regular column.

The application for the large-scale preparation of sulfinic ester attracts our attentions. We performed the gram-scale synthesis with diphenyl disulfide (1a) and ethanol (2a) as starting materials under the standard reaction conditions (Scheme 2). It was found that the reaction could still complete within $4 \mathrm{~h}$, providing the product $\mathbf{3 a}$ in almost the same level of yield (1.19 g, 87\%). Excellent yield, mild conditions and operational convenience, bode well for wide spread application of this methodology for large-scale preparation of sulfinic esters.

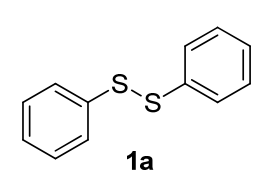

$873.3 \mathrm{mg}, 4 \mathrm{mmol}$

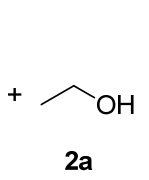

$2 a$

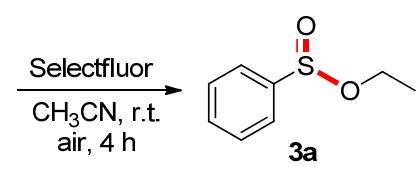

$1.19 \mathrm{~g}, 87 \%$ yield
Scheme 2 Gram-scale synthesis

We also tried to extend the current system to thiol for the preparation of sulfinic ester. When 4-chlorobenzenethiol (4) was subjected to this system under the standard conditions, the reaction could also happen and afforded the desired sulfinic ester $3 f$ in $83 \%$ yield (Scheme 3 ).

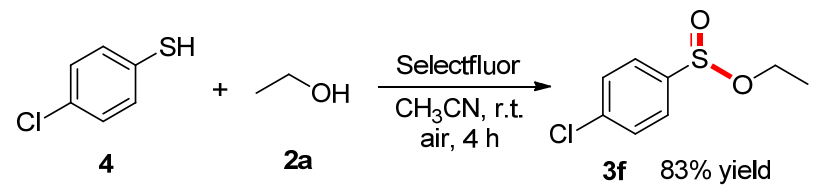

Scheme 3 Reaction of thiol

Based on the above experimental results and literature reports, ${ }^{[9,15-17]}$ a plausible mechanism for this Selectfluorpromoted oxidative reaction was proposed in Scheme 4. Initially, disulfide 1a reacts with Selectfluor to form a sulfonium intermediate $\mathbf{A},{ }^{[15-16]}$ which reacts with oxygen to give the thiosulfinate intermediate B. Subsequently, a second similar oxidation happens with the aid of Selectfluor to form the disulfoxide $\mathbf{C}$. Substitution of intermediate $\mathbf{C}$ via the nucleophilic attack by ethanol generates the sulfinic $\mathbf{3 a}$ with the release of sulfinyl anion D. On the other hand, sulfinyl anion D reacts with Selectfluor to give the sulfinyl fluoride $\mathbf{E}$, which is transferred into the second sulfinic ester 3a via the substitution by ethanol (path a). The reaction conducted under nitrogen atomosphere also afforded the corresponding sulfinic ester, which indicates that alcohol may also act as the oxygen source during this transformation. Thus, initial generation of sulfonium intermediate A, subsequent substitution by ethanol to give intermediate $\mathbf{G}$ and $\mathrm{O}-\mathrm{H} / \mathrm{O}-\mathrm{C}$ bond cleavage to give the thiosulfinate intermediate $\mathbf{B}$ could not be excluded (path b).

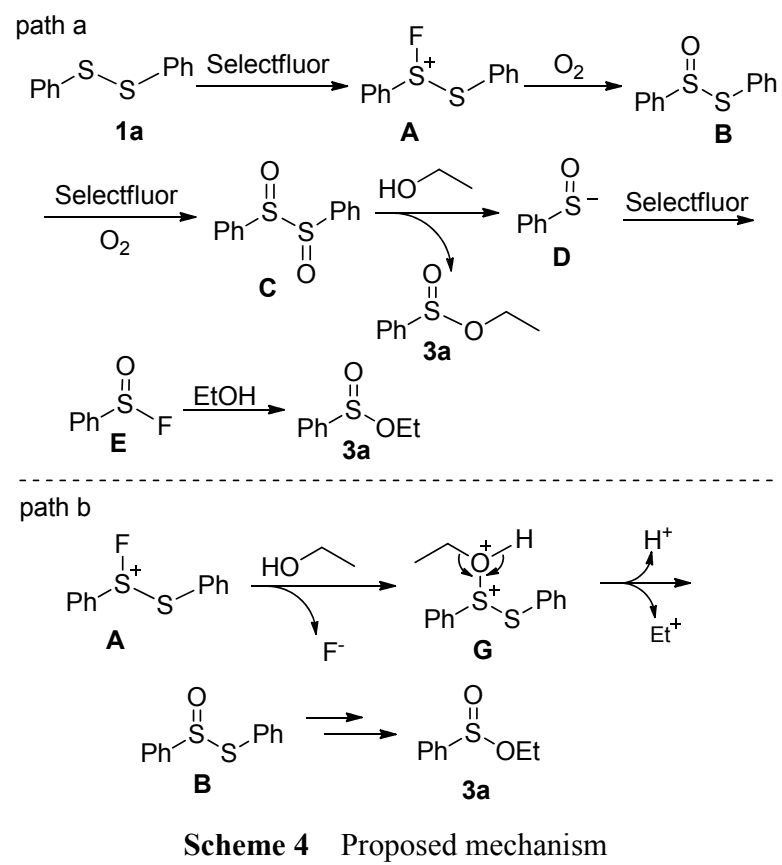

\section{Conclusions}

In summary, a new and efficient Selectfluor-promoted oxidation reaction of diaryldisulfides and alcohols was developed. The reaction was carried out under mild conditions and tolerated a wide range of alcohol substrates, even 
tertiary and natural alcohols, resulting in the unexpected sulfinic esters in excellent yields. This reaction provides a new strategy for the synthesis of sulfinic esters.

\section{Experimental section}

\subsection{General information}

NMR spectra were recorded on Bruker $600 \mathrm{MHz}$ and 400 $\mathrm{MHz}$ spectrometers. Mass spectra (MS) were measured on a Shimadzu LCMS-2020 with an etrospray ionization (ESI) probe operating in positive mode. High resolution mass spectra (HRMS) were measured on a Agilent 6210 ESI/ TOF MS instrument. All the commercial reagents including solvents were used directly without further purification. All the experiments were monitored by thin layer chromatography (TLC) with UV light. The TLC employed $0.25 \mathrm{~mm}$ silica gel coated on glass plates. Column chromatography was performed with silica gel 60 (300 400 mesh).

\subsection{Reaction of various disulfides and alcohols}

Into a $10 \mathrm{~mL}$ vial disulfide $1(0.2 \mathrm{mmol})$, alcohol 2 (1 $\mathrm{mL})$, Selectfluor (4 equiv.) and acetonitrile $(4 \mathrm{~mL})$ were added. The mixture was stirred under air at room temperature for $4 \mathrm{~h}$. Then, the reaction was diluted with $\mathrm{H}_{2} \mathrm{O}(20$ $\mathrm{mL})$ and extracted with EtOAc $(20 \mathrm{~mL} \times 3)$. The combined organic layers were dried with anhydrous $\mathrm{Na}_{2} \mathrm{SO}_{4}$, filtered and concentrated in vacuo. The residue was purified by column chromatography using hexane/EtOAc $(V: V=$ $10: 1$ ) as eluent to afford the desired product 3 .

Ethyl benzenesulfinate (3a): ${ }^{[9 a]}$ colorless oil, 94\% yield. ${ }^{1} \mathrm{H}$ NMR $\left(600 \mathrm{MHz}, \mathrm{CDCl}_{3}\right) \delta: 7.74 \sim 7.73(\mathrm{~m}, 2 \mathrm{H}), 7.57 \sim$ $7.54(\mathrm{~m}, 3 \mathrm{H}), 4.16 \sim 4.11(\mathrm{~m}, 1 \mathrm{H}), 3.78 \sim 3.72(\mathrm{~m}, 1 \mathrm{H}), 1.31$ (t, $J=7.08 \mathrm{~Hz}, 3 \mathrm{H})$; IR (ATR) $v: 3059,2981,1444,1129$, $878 \mathrm{~cm}^{-1}$; MS (ESI) $\mathrm{m} / \mathrm{z}: 171.2[\mathrm{M}+\mathrm{H}]^{+}$.

Ethyl 4-methylbenzenesulfinate $(\mathbf{3 b}):{ }^{[9 a]}$ colorless oil, $86 \%$ yield. ${ }^{1} \mathrm{H}$ NMR $\left(600 \mathrm{MHz}, \mathrm{CDCl}_{3}\right) \delta: 7.63(\mathrm{~d}, J=8.16$ $\mathrm{Hz}, 2 \mathrm{H}), 7.36$ (d, J=7.92 Hz, 2H), 4.14 4.09 (m, 1H), $3.77 \sim 3.72(\mathrm{~m}, 1 \mathrm{H}), 2.45(\mathrm{~s}, 3 \mathrm{H}), 1.31(\mathrm{t}, J=7.11 \mathrm{~Hz}, 3 \mathrm{H})$; IR (ATR) v: 2980, 2922, 1596, 1132, $881 \mathrm{~cm}^{-1}$; MS (ESI) $\mathrm{m} / \mathrm{z}: 185.0[\mathrm{M}+\mathrm{H}]^{+}$.

Ethyl 4-methoxybenzenesulfinate $(\mathbf{3 c}):^{[10 \mathrm{a}]}$ yellow oil, $71 \%$ yield. ${ }^{1} \mathrm{H}$ NMR $\left(600 \mathrm{MHz}, \mathrm{CDCl}_{3}\right) \delta: 7.65(\mathrm{~d}, J=8.82$ $\mathrm{Hz}, 2 \mathrm{H}), 7.02(\mathrm{~d}, J=8.82 \mathrm{~Hz}, 2 \mathrm{H}), 4.11 \sim 4.06(\mathrm{~m}, 1 \mathrm{H})$, $3.86(\mathrm{~s}, 3 \mathrm{H}), 3.75 \sim 3.69(\mathrm{~m}, 1 \mathrm{H}), 1.28(\mathrm{t}, J=7.08 \mathrm{~Hz}, 3 \mathrm{H})$; IR (ATR) $v: 2979,1593,1129,881 \mathrm{~cm}^{-1}$; MS (ESI) $\mathrm{m} / \mathrm{z}$ : $200.9[\mathrm{M}+\mathrm{H}]^{+}$

Ethyl 2-fluorobenzenesulfinate (3d): yellow oil, $88 \%$ yield. ${ }^{1} \mathrm{H} \mathrm{NMR}\left(600 \mathrm{MHz}, \mathrm{CDCl}_{3}\right) \delta: 7.87 \sim 7.84(\mathrm{~m}, 1 \mathrm{H})$, $7.56 \sim 7.52(\mathrm{~m}, 1 \mathrm{H}), 7.35 \sim 7.33(\mathrm{~m}, 1 \mathrm{H}), 7.16 \sim 7.13(\mathrm{~m}$, $1 \mathrm{H}), 4.20 \sim 4.15(\mathrm{~m}, 1 \mathrm{H}), 3.87 \sim 3.81(\mathrm{~m}, 1 \mathrm{H}), 1.32(\mathrm{t}, J=$ $7.11 \mathrm{~Hz}, 3 \mathrm{H}) ;{ }^{13} \mathrm{C}$ NMR $\left(150 \mathrm{MHz}, \mathrm{CDCl}_{3}\right) \delta: 160.5(\mathrm{~d}, J=$ $250.0 \mathrm{~Hz}), 134.2(\mathrm{~d}, J=8.6 \mathrm{~Hz}), 132.1(\mathrm{~d}, J=15.8 \mathrm{~Hz})$, $126.3(\mathrm{~d}, J=2.3 \mathrm{~Hz}), 124.6(\mathrm{~d}, J=3.7 \mathrm{~Hz}), 116.2(\mathrm{~d}, J=$ $20.3 \mathrm{~Hz}), 62.4,15.5 ;{ }^{19} \mathrm{~F}$ NMR $\left(565 \mathrm{MHz}, \mathrm{CDCl}_{3}\right) \delta$ : -115.6 ; IR (ATR) $v: 2983,1597,1259,1135,880 \mathrm{~cm}^{-1}$; HRMS (ESI) calcd for $\mathrm{C}_{8} \mathrm{H}_{10} \mathrm{FO}_{2} \mathrm{~S}[\mathrm{M}+\mathrm{H}]^{+}$189.0380, found 189.0386 .

Ethyl 3-fluorobenzenesulfinate (3e): yellow oil, 89\% yield. ${ }^{1} \mathrm{H} \mathrm{NMR}\left(600 \mathrm{MHz}, \mathrm{CDCl}_{3}\right) \delta: 7.55 \sim 7.51(\mathrm{~m}, 1 \mathrm{H})$, $7.50 \sim 7.49(\mathrm{~m}, 1 \mathrm{H}), 7.46 \sim 7.44(\mathrm{~m}, 1 \mathrm{H}), 7.26 \sim 7.23(\mathrm{~m}$, $1 \mathrm{H}), 4.17 \sim 4.11(\mathrm{~m}, 1 \mathrm{H}), 3.77 \sim 3.72(\mathrm{~m}, 1 \mathrm{H}), 1.31(\mathrm{t}, J=$ $7.08 \mathrm{~Hz}, 3 \mathrm{H}) ;{ }^{13} \mathrm{C} \mathrm{NMR}\left(150 \mathrm{MHz}, \mathrm{CDCl}_{3}\right) \delta: 163.7$ (d, $J=$ $250.4 \mathrm{~Hz}), 147.3(\mathrm{~d}, J=5.1 \mathrm{~Hz}), 130.8$ (d, $J=7.4 \mathrm{~Hz}), 121.1$ $(\mathrm{d}, J=3.3 \mathrm{~Hz}), 119.3(\mathrm{~d}, J=21.4 \mathrm{~Hz}), 112.6(\mathrm{~d}, J=23.5$ $\mathrm{Hz}), 61.4,15.5 ;{ }^{19} \mathrm{~F}$ NMR $\left(565 \mathrm{MHz}, \mathrm{CDCl}_{3}\right) \delta:-110.0$; IR (ATR) $v: 2979,1593,1253,1128,880 \mathrm{~cm}^{-1}$; HRMS (ESI) calcd for $\mathrm{C}_{8} \mathrm{H}_{9} \mathrm{FNaO}_{2} \mathrm{~S}[\mathrm{M}+\mathrm{Na}]^{+}$211.0199, found 211.0196.

Ethyl 4-chlorobenzenesulfinate (3f): ${ }^{[10 c]}$ yellow oil, 95\% yield. ${ }^{1} \mathrm{H} \mathrm{NMR}\left(600 \mathrm{MHz}, \mathrm{CDCl}_{3}\right) \delta: 7.66(\mathrm{~d}, J=8.58 \mathrm{~Hz}$, $2 \mathrm{H}), 7.52(\mathrm{~d}, J=8.64 \mathrm{~Hz}, 2 \mathrm{H}), 4.14 \sim 4.09(\mathrm{~m}, 1 \mathrm{H}), 3.76 \sim$ $3.71(\mathrm{~m}, 1 \mathrm{H}), 1.30(\mathrm{t}, J=7.11 \mathrm{~Hz}, 3 \mathrm{H})$; MS (ESI) calcd for $\mathrm{C}_{8} \mathrm{H}_{10} \mathrm{ClO}_{2} \mathrm{~S}[\mathrm{M}+\mathrm{H}]^{+}$205.0, found 204.9.

Ethyl 3,5-dichlorobenzenesulfinate (3g): yellow oil, 98\% yield. ${ }^{1} \mathrm{H} \mathrm{NMR}\left(600 \mathrm{MHz}, \mathrm{CDCl}_{3}\right) \delta: 7.58(\mathrm{~d}, J=1.86 \mathrm{~Hz}$, $2 \mathrm{H}), 7.52 \sim 7.51(\mathrm{~m}, 1 \mathrm{H}), 4.18 \sim 4.13(\mathrm{~m}, 1 \mathrm{H}), 3.79 \sim 3.74$ $(\mathrm{m}, 1 \mathrm{H}), 1.34(\mathrm{t}, J=7.11 \mathrm{~Hz}, 3 \mathrm{H}) ;{ }^{13} \mathrm{C} \mathrm{NMR}(150 \mathrm{MHz}$, $\mathrm{CDCl}_{3}$ ) $\delta: 148.1,136.1,132.1,123.8,61.9,15.5$; IR (ATR) $v: 2976,1593,1130,870 \mathrm{~cm}^{-1}$; HRMS (ESI) calcd for $\mathrm{C}_{8} \mathrm{H}_{9} \mathrm{Cl}_{2} \mathrm{O}_{2} \mathrm{~S}[\mathrm{M}+\mathrm{H}]^{+}$238.9695, found 238.9699.

Ethyl 3-nitrobenzenesulfinate (3h): yellow oil, 91\% yield. ${ }^{1} \mathrm{H}$ NMR $\left(600 \mathrm{MHz}, \mathrm{CDCl}_{3}\right) \delta: 8.55(\mathrm{t}, J=1.86 \mathrm{~Hz}$, $1 \mathrm{H}), 8.41 \sim 8.39(\mathrm{~m}, 1 \mathrm{H}), 8.06 \sim 8.05(\mathrm{~m}, 1 \mathrm{H}), 7.79(\mathrm{t}, J=$ $7.92 \mathrm{~Hz}, 1 \mathrm{H}), 4.22 \sim 4.17(\mathrm{~m}, 1 \mathrm{H}), 3.84 \sim 3.79(\mathrm{~m}, 1 \mathrm{H})$, $1.34(\mathrm{t}, J=7.11 \mathrm{~Hz}, 3 \mathrm{H}) ;{ }^{13} \mathrm{C}$ NMR $\left(150 \mathrm{MHz}, \mathrm{CDCl}_{3}\right) \delta$ : $148.5,147.4,131.1,130.4,126.7,120.7,62.4,15.6$; IR (ATR) $v: 3094,2985,1530,1137,875 \mathrm{~cm}^{-1}$; HRMS (ESI) calcd for $\mathrm{C}_{8} \mathrm{H}_{10} \mathrm{NO}_{4} \mathrm{~S}[\mathrm{M}+\mathrm{H}]^{+}$216.0325, found 216.0329 .

Ethyl pyridine-2-sulfinate (3i): yellow oil, $75 \%$ yield. ${ }^{1} \mathrm{H}$ NMR $\left(600 \mathrm{MHz}, \mathrm{CDCl}_{3}\right) \delta: 8.70(\mathrm{~d}, J=4.44 \mathrm{~Hz}, 1 \mathrm{H}), 8.00$ $(\mathrm{d}, J=7.80 \mathrm{~Hz}, 1 \mathrm{H}), 7.96 \sim 7.93(\mathrm{~m}, 1 \mathrm{H}), 7.47 \sim 7.45(\mathrm{~m}$, $1 \mathrm{H}), 4.22 \sim 4.17(\mathrm{~m}, 1 \mathrm{H}), 3.84 \sim 3.79(\mathrm{~m}, 1 \mathrm{H}), 1.31(\mathrm{t}, J=$ $7.08 \mathrm{~Hz}, 3 \mathrm{H}) ;{ }^{13} \mathrm{C} \mathrm{NMR}\left(150 \mathrm{MHz}, \mathrm{CDCl}_{3}\right) \delta: 163.7,150.0$, 137.9, 126.2, 119.8, 62.6, 15.6; IR (ATR) $v: 3093,1612$, 1451, 1240, 1150, $1042 \mathrm{~cm}^{-1}$; HRMS (ESI) calcd for $\mathrm{C}_{7} \mathrm{H}_{9} \mathrm{NNaO}_{2} \mathrm{~S}[\mathrm{M}+\mathrm{Na}]^{+}$194.0246, found 194.0252 .

Ethyl thiophene-2-sulfinate $(\mathbf{3 j}$ ): yellow oil, $83 \%$ yield. ${ }^{1} \mathrm{H}$ NMR $\left(600 \mathrm{MHz}, \mathrm{CDCl}_{3}\right) \delta: 7.66(\mathrm{~d}, J=4.86 \mathrm{~Hz}, 1 \mathrm{H})$, $7.51(\mathrm{~d}, J=3.66 \mathrm{~Hz}, 1 \mathrm{H}), 7.18(\mathrm{t}, J=4.29 \mathrm{~Hz}, 1 \mathrm{H}), 4.25 \sim$ $4.20(\mathrm{~m}, 1 \mathrm{H}), 3.92 \sim 3.87(\mathrm{~m}, 1 \mathrm{H}), 1.36(\mathrm{t}, J=7.08 \mathrm{~Hz}, 3 \mathrm{H})$; ${ }^{13} \mathrm{C}$ NMR $\left(150 \mathrm{MHz}, \mathrm{CDCl}_{3}\right) \delta: 147.9,131.4,129.7,127.7$, $60.8,15.4$; IR (ATR) v: 3098, 1535, 1135, $870 \mathrm{~cm}^{-1}$; HRMS (ESI) calcd for $\mathrm{C}_{6} \mathrm{H}_{8} \mathrm{NaO}_{2} \mathrm{~S}_{2}[\mathrm{M}+\mathrm{Na}]^{+}$198.9858, found 198.9861 .

Ethyl 1-phenyl-1H-tetrazole-5-sulfinate (3k): yellow oil, $30 \%$ yield. ${ }^{1} \mathrm{H}$ NMR $\left(600 \mathrm{MHz}, \mathrm{CDCl}_{3}\right) \delta: 7.65 \sim 7.62(\mathrm{~m}$, $5 \mathrm{H}), 4.46 \sim 4.41(\mathrm{~m}, 1 \mathrm{H}), 4.19 \sim 4.14(\mathrm{~m}, 1 \mathrm{H}), 1.33(\mathrm{t}, J=$ $7.08 \mathrm{~Hz}, 3 \mathrm{H}) ;{ }^{13} \mathrm{C} \mathrm{NMR}\left(150 \mathrm{MHz}, \mathrm{CDCl}_{3}\right) \delta: 157.3,133.3$, 131.1, 129.9, 124.9, 64.9, 15.3; IR (ATR) v: 3066, 2924, 1597, 1207, 1091, $997 \mathrm{~cm}^{-1}$; HRMS (ESI) calcd for $\mathrm{C}_{9} \mathrm{H}_{10} \mathrm{~N}_{4} \mathrm{NaO}_{2} \mathrm{~S}[\mathrm{M}+\mathrm{Na}]^{+}$261.0417, found 261.0413.

Methyl benzenesulfinate (31): ${ }^{[9 \mathrm{a}]}$ colorless oil, $94 \%$ yield. ${ }^{1} \mathrm{H}$ NMR $\left(600 \mathrm{MHz}, \mathrm{CDCl}_{3}\right) \delta: 7.74 \sim 7.72(\mathrm{~m}, 2 \mathrm{H}), 7.58 \sim$ $7.56(\mathrm{~m}, 3 \mathrm{H}), 3.50(\mathrm{~s}, 3 \mathrm{H})$; MS (ESI) $m / z: 156.9[\mathrm{M}+\mathrm{H}]^{+}$.

Propyl benzenesulfinate $(\mathbf{3 m}):^{[9]}$ colorless oil, $96 \%$ 
yield. ${ }^{1} \mathrm{H}$ NMR $\left(600 \mathrm{MHz}, \mathrm{CDCl}_{3}\right) \delta: 7.73 \sim 7.72(\mathrm{~m}, 2 \mathrm{H})$, $7.56 \sim 7.54(\mathrm{~m}, 3 \mathrm{H}), 4.04 \sim 4.00(\mathrm{~m}, 1 \mathrm{H}), 3.62 \sim 3.58(\mathrm{~m}$, $1 \mathrm{H}), 1.70 \sim 1.64(\mathrm{~m}, 2 \mathrm{H}), 0.94(\mathrm{t}, J=7.41 \mathrm{~Hz}, 3 \mathrm{H}) ; \mathrm{MS}$ (ESI) $m / z: 185.1[\mathrm{M}+\mathrm{H}]^{+}$.

Butyl benzenesulfinate (3n): ${ }^{[9 a]}$ colorless oil, 98\% yield. ${ }^{1} \mathrm{H}$ NMR $\left(600 \mathrm{MHz}, \mathrm{CDCl}_{3}\right) \delta: 7.72 \sim 7.71(\mathrm{~m}, 2 \mathrm{H}), 7.55 \sim$ $7.53(\mathrm{~m}, 3 \mathrm{H}), 4.07 \sim 4.03(\mathrm{~m}, 1 \mathrm{H}), 3.65 \sim 3.61(\mathrm{~m}, 1 \mathrm{H})$, $1.64 \sim 1.59(\mathrm{~m}, 2 \mathrm{H}), 1.39 \sim 1.33(\mathrm{~m}, 2 \mathrm{H}), 0.90(\mathrm{t}, J=7.41$ $\mathrm{Hz}, 3 \mathrm{H})$; MS (ESI) $m / z: 199.1[\mathrm{M}+\mathrm{H}]^{+}$.

Pentyl benzenesulfinate (3o) $:^{[8 b]}$ colorless oil, 95\% yield. ${ }^{1} \mathrm{H}$ NMR $\left(600 \mathrm{MHz}, \mathrm{CDCl}_{3}\right) \delta: 7.72 \sim 7.70(\mathrm{~m}, 2 \mathrm{H}), 7.55 \sim$ $7.52(\mathrm{~m}, 3 \mathrm{H}), 4.06 \sim 4.02(\mathrm{~m}, 1 \mathrm{H}), 3.63 \sim 3.59(\mathrm{~m}, 1 \mathrm{H})$, $1.65 \sim 1.61(\mathrm{~m}, 2 \mathrm{H}), 1.31 \sim 1.26(\mathrm{~m}, 4 \mathrm{H}), 0.88(\mathrm{t}, J=7.11$ $\mathrm{Hz}, 3 \mathrm{H})$; MS (ESI) $m / z: 213.0[\mathrm{M}+\mathrm{H}]^{+}$.

Isopropyl benzenesulfinate $(\mathbf{3 p}):{ }^{[9 \mathrm{a}]}$ colorless oil, $98 \%$ yield. ${ }^{1} \mathrm{H}$ NMR $\left(600 \mathrm{MHz}, \mathrm{CDCl}_{3}\right) \delta: 7.73 \sim 7.71(\mathrm{~m}, 2 \mathrm{H})$, $7.55 \sim 7.52(\mathrm{~m}, 3 \mathrm{H}), 4.65 \sim 4.59(\mathrm{~m}, 1 \mathrm{H}), 1.40(\mathrm{~d}, J=6.24$ $\mathrm{Hz}, 3 \mathrm{H}), 1.27$ (d, $J=6.30 \mathrm{~Hz}, 3 \mathrm{H})$; MS (ESI) $m / z: 185.0$ $[\mathrm{M}+\mathrm{H}]^{+}$.

Isobutyl benzenesulfinate $(\mathbf{3 q}):{ }^{[9 \mathrm{a}]}$ colorless oil, $88 \%$ yield. ${ }^{1} \mathrm{H}$ NMR $\left(600 \mathrm{MHz}, \mathrm{CDCl}_{3}\right) \delta: 7.73 \sim 7.71(\mathrm{~m}, 2 \mathrm{H})$, $7.56 \sim 7.53(\mathrm{~m}, 3 \mathrm{H}), 3.84(\mathrm{dd}, J=6.60,9.60 \mathrm{~Hz}, 1 \mathrm{H}), 3.38$ $(\mathrm{dd}, J=6.54,9.60 \mathrm{~Hz}, 1 \mathrm{H}), 1.95 \sim 1.88(\mathrm{~m}, 1 \mathrm{H}), 0.92(\mathrm{~d}$, $J=6.72 \mathrm{~Hz}, 3 \mathrm{H}), 0.91(\mathrm{~d}, J=6.72 \mathrm{~Hz}, 3 \mathrm{H})$; MS (ESI) $m / z$ : $199.0[\mathrm{M}+\mathrm{H}]^{+}$.

Isopentyl benzenesulfinate (3r): colorless oil, 91\% yield. ${ }^{1} \mathrm{H}$ NMR $\left(600 \mathrm{MHz}, \mathrm{CDCl}_{3}\right) \delta: 7.72 \sim 7.71(\mathrm{~m}, 2 \mathrm{H}), 7.55 \sim$ $7.53(\mathrm{~m}, 3 \mathrm{H}), 4.10 \sim 4.06(\mathrm{~m}, 1 \mathrm{H}), 3.67 \sim 3.63(\mathrm{~m}, 1 \mathrm{H})$, $1.72 \sim 1.65(\mathrm{~m}, 1 \mathrm{H}), 1.54 \sim 1.50(\mathrm{~m}, 2 \mathrm{H}), 0.89(\mathrm{~d}, J=6.66$ $\mathrm{Hz}, 3 \mathrm{H}), 0.85(\mathrm{~d}, J=6.72 \mathrm{~Hz}, 3 \mathrm{H}) ;{ }^{13} \mathrm{C}$ NMR $(150 \mathrm{MHz}$, $\left.\mathrm{CDCl}_{3}\right) \delta: 144.8,132.0,129.0,125.2,63.2,38.4,24.7,22.3$, 22.2; IR (ATR) v: 2933, 1445, 1132, 1007, $845 \mathrm{~cm}^{-1}$; HRMS (ESI) calcd for $\mathrm{C}_{11} \mathrm{H}_{16} \mathrm{NaO}_{2} \mathrm{~S}[\mathrm{M}+\mathrm{Na}]^{+}$235.0763, found 235.0768 .

Tert-butyl benzenesulfinate $(3 \mathrm{~s}):{ }^{[8 \mathrm{~b}]}$ colorless oil, $57 \%$ yield. ${ }^{1} \mathrm{H} \mathrm{NMR}\left(600 \mathrm{MHz}, \mathrm{CDCl}_{3}\right) \delta: 7.71 \sim 7.69(\mathrm{~m}, 2 \mathrm{H})$, $7.54 \sim 7.52(\mathrm{~m}, 3 \mathrm{H}), 1.58(\mathrm{~s}, 9 \mathrm{H})$; MS (ESI) calcd for $\mathrm{C}_{10} \mathrm{H}_{14} \mathrm{NaO}_{2} \mathrm{~S}[\mathrm{M}+\mathrm{Na}]^{+}$221.1, found 221.0.

Cyclobutyl benzenesulfinate (3t): colorless oil, 98\% yield. ${ }^{1} \mathrm{H} \mathrm{NMR}\left(600 \mathrm{MHz}, \mathrm{CDCl}_{3}\right) \delta: 7.72 \sim 7.71(\mathrm{~m}, 2 \mathrm{H})$, $7.54 \sim 7.51(\mathrm{~m}, 3 \mathrm{H}), 4.75 \sim 4.70(\mathrm{~m}, 1 \mathrm{H}), 2.36 \sim 2.33(\mathrm{~m}$, $1 \mathrm{H}), 2.27 \sim 2.20(\mathrm{~m}, 1 \mathrm{H}), 2.09 \sim 2.02(\mathrm{~m}, 1 \mathrm{H}), 1.96 \sim 1.89$ $(\mathrm{m}, 1 \mathrm{H}), 1.75 \sim 1.70(\mathrm{~m}, 1 \mathrm{H}), 1.54 \sim 1.47(\mathrm{~m}, 1 \mathrm{H}) ;{ }^{13} \mathrm{C}$ NMR $\left(150 \mathrm{MHz}, \mathrm{CDCl}_{3}\right) \delta: 145.3,132.0,129.0,125.1$, 70.1, 32.1, 32.0, 13.4; IR (ATR) v: 2984, 1444, 1136, 1047, 924, $790 \mathrm{~cm}^{-1}$; HRMS (ESI) calcd for $\mathrm{C}_{10} \mathrm{H}_{12} \mathrm{NaO}_{2} \mathrm{~S}[\mathrm{M}+$ $\mathrm{Na}]^{+} 219.0450$, found 219.0456 .

Cyclopentyl benzenesulfinate $(3 \mathbf{u}):{ }^{[8 \mathrm{a}]}$ colorless oil, $80 \%$ yield. ${ }^{1} \mathrm{H}$ NMR $\left(600 \mathrm{MHz}, \mathrm{CDCl}_{3}\right) \delta: 7.72 \sim 7.70(\mathrm{~m}, 2 \mathrm{H})$, $7.54 \sim 7.52(\mathrm{~m}, 3 \mathrm{H}), 4.86 \sim 4.83(\mathrm{~m}, 1 \mathrm{H}), 1.92 \sim 1.89(\mathrm{~m}$, $2 \mathrm{H}), 1.77 \sim 1.69(\mathrm{~m}, 4 \mathrm{H}), 1.62 \sim 1.51(\mathrm{~m}, 2 \mathrm{H})$; IR (ATR) $v$ : $3059,2828,1445,1123,994,757 \mathrm{~cm}^{-1}$; MS (ESI) calcd for $\mathrm{C}_{11} \mathrm{H}_{15} \mathrm{O}_{2} \mathrm{~S}[\mathrm{M}+\mathrm{H}]^{+}$211.1, found 211.1.

Cyclohexyl benzenesulfinate $(3 \mathrm{v}):{ }^{[9 \mathrm{a}]}$ colorless oil, $84 \%$ yield. ${ }^{1} \mathrm{H} \mathrm{NMR}\left(600 \mathrm{MHz}, \mathrm{CDCl}_{3}\right) \delta: 7.74 \sim 7.72(\mathrm{~m}, 2 \mathrm{H})$, $7.55 \sim 7.52(\mathrm{~m}, 3 \mathrm{H}), 4.38 \sim 4.34(\mathrm{~m}, 1 \mathrm{H}), 2.05 \sim 2.02(\mathrm{~m}$, $1 \mathrm{H}), 1.83 \sim 1.71(\mathrm{~m}, 3 \mathrm{H}), 1.64 \sim 1.58(\mathrm{~m}, 1 \mathrm{H}), 1.53 \sim 1.49$ $(\mathrm{m}, 2 \mathrm{H}), 1.41 \sim 1.35(\mathrm{~m}, 1 \mathrm{H}), 1.33 \sim 1.23(\mathrm{~m}, 2 \mathrm{H}) ; \mathrm{IR}$ (ATR) $v$ : 2954, 1698, 1446, 1121, 1011, 991, $753 \mathrm{~cm}^{-1}$; MS (ESI) calcd for $\mathrm{C}_{12} \mathrm{H}_{17} \mathrm{O}_{2} \mathrm{~S}[\mathrm{M}+\mathrm{H}]^{+}$225.1, found 225.2.

Cycloheptyl benzenesulfinate $(\mathbf{3 w}):{ }^{[8 \mathrm{~b}]}$ colorless oil, $87 \%$ yield. ${ }^{1} \mathrm{H}$ NMR $\left(600 \mathrm{MHz}, \mathrm{CDCl}_{3}\right) \delta: 7.73 \sim 7.71(\mathrm{~m}, 2 \mathrm{H})$, $7.54 \sim 7.52(\mathrm{~m}, 3 \mathrm{H}), 4.55 \sim 4.51(\mathrm{~m}, 1 \mathrm{H}), 2.09 \sim 2.04(\mathrm{~m}$, $1 \mathrm{H}), 1.89 \sim 1.84(\mathrm{~m}, 2 \mathrm{H}), 1.74 \sim 1.69(\mathrm{~m}, 2 \mathrm{H}), 1.65 \sim 1.61$ $(\mathrm{m}, 1 \mathrm{H}), 1.56 \sim 1.52(\mathrm{~m}, 4 \mathrm{H}), 1.49 \sim 1.44(\mathrm{~m}, 1 \mathrm{H}), 1.39 \sim$ $1.34(\mathrm{~m}, 1 \mathrm{H})$; MS (ESI) calcd for $\mathrm{C}_{13} \mathrm{H}_{19} \mathrm{O}_{2} \mathrm{~S}[\mathrm{M}+\mathrm{H}]^{+}$ 239.1, found 239.1.

Cyclododecyl benzenesulfinate $(\mathbf{3 x})$ : colorless oil, $70 \%$ yield. ${ }^{1} \mathrm{H} \mathrm{NMR}\left(600 \mathrm{MHz}, \mathrm{CDCl}_{3}\right) \delta: 7.75 \sim 7.73(\mathrm{~m}, 2 \mathrm{H})$, $7.55 \sim 7.53(\mathrm{~m}, 3 \mathrm{H}), 4.54 \sim 4.50(\mathrm{~m}, 1 \mathrm{H}), 1.89 \sim 1.84(\mathrm{~m}$, $1 \mathrm{H}), 1.77 \sim 1.68(\mathrm{~m}, 2 \mathrm{H}), 1.53 \sim 1.48(\mathrm{~m}, 2 \mathrm{H}), 1.44 \sim 1.41$ $(\mathrm{m}, 3 \mathrm{H}), 1.38 \sim 1.26(\mathrm{~m}, 14 \mathrm{H}) ;{ }^{13} \mathrm{C}$ NMR $(150 \mathrm{MHz}$, $\left.\mathrm{CDCl}_{3}\right) \delta: 145.9,131.9,128.9,125.0,78.4,30.9,30.8,24.2$, 24.0, 23.8, 23.4, 23.3, 23.2, 23.1, 20.9, 20.8; IR (ATR) v: 2955, 2869, 1447, 1136, 955, $851 \mathrm{~cm}^{-1}$; HRMS (ESI) calcd for $\mathrm{C}_{18} \mathrm{H}_{28} \mathrm{NaO}_{2} \mathrm{~S}[\mathrm{M}+\mathrm{Na}]^{+}$331.1702, found 331.1708.

$(3 r, 5 r, 7 r)$-Adamantan-1-ylmethyl benzenesulfinate (3y): colorless oil, $86 \%$ yield. ${ }^{1} \mathrm{H}$ NMR $\left(600 \mathrm{MHz}, \mathrm{CDCl}_{3}\right) \delta$ : $7.71 \sim 7.69(\mathrm{~m}, 2 \mathrm{H}), 7.55 \sim 7.51(\mathrm{~m}, 3 \mathrm{H}), 3.61(\mathrm{~d}, J=9.54$ $\mathrm{Hz}, 1 \mathrm{H}), 3.09$ (d, $J=9.54 \mathrm{~Hz}, 1 \mathrm{H}), 1.96(\mathrm{~s}, 3 \mathrm{H}), 1.71 \sim 1.69$ $(\mathrm{m}, 3 \mathrm{H}), 1.62 \sim 1.60(\mathrm{~m}, 3 \mathrm{H}), 1.51 \sim 1.46(\mathrm{~m}, 6 \mathrm{H}) ;{ }^{13} \mathrm{C}$ NMR $\left(150 \mathrm{MHz}, \mathrm{CDCl}_{3}\right) \delta: 144.8,131.9,129.0,125.4$, 73.8, 39.2, 36.9, 33.5, 28.0; IR (ATR) $v$ : 2926, 2848, 1460, $1102,968,845 \mathrm{~cm}^{-1}$; HRMS (ESI) calcd for $\mathrm{C}_{17} \mathrm{H}_{22} \mathrm{NaO}_{2} \mathrm{~S}$ $[\mathrm{M}+\mathrm{Na}]^{+}$313.1233, found 313.1237.

Ethyl 2-((phenylsulfinyl)oxy)acetate (3z): colorless oil, $46 \%$ yield. ${ }^{1} \mathrm{H}$ NMR $\left(600 \mathrm{MHz}, \mathrm{CDCl}_{3}\right) \delta: 7.84(\mathrm{~d}, J=7.62$ $\mathrm{Hz}, 2 \mathrm{H}), 7.61 \sim 7.56(\mathrm{~m}, 3 \mathrm{H}), 4.60(\mathrm{~d}, J=16.2 \mathrm{~Hz}, 1 \mathrm{H})$, $4.25 \sim 4.20(\mathrm{~m}, 3 \mathrm{H}), 1.29(\mathrm{t}, J=7.14 \mathrm{~Hz}, 3 \mathrm{H}) ;{ }^{13} \mathrm{C} \mathrm{NMR}$ $\left(150 \mathrm{MHz}, \mathrm{CDCl}_{3}\right) \delta: 168.1,144.1,132.6,129.1,125.5$, 61.7, 60.4, 14.1; IR (ATR) v: 2984, 1737, 1444, 1207, 1093, $757 \mathrm{~cm}^{-1}$; HRMS (ESI) calcd for $\mathrm{C}_{10} \mathrm{H}_{13} \mathrm{O}_{4} \mathrm{~S}[\mathrm{M}+\mathrm{H}]^{+}$ 229.0529, found 229.0531.

Phenethyl benzenesulfinate (3aa): ${ }^{[8 a]}$ colorless oil, $78 \%$ yield. ${ }^{1} \mathrm{H}$ NMR $\left(600 \mathrm{MHz}, \mathrm{CDCl}_{3}\right) \delta: 7.65 \sim 7.64(\mathrm{~m}, 2 \mathrm{H})$, $7.57 \sim 7.50(\mathrm{~m}, 3 \mathrm{H}), 7.32 \sim 7.30(\mathrm{~m}, 2 \mathrm{H}), 7.28 \sim 7.25(\mathrm{~m}$, $1 \mathrm{H}), 7.18 \sim 7.17(\mathrm{~m}, 2 \mathrm{H}), 4.31 \sim 4.27(\mathrm{~m}, 1 \mathrm{H}), 3.87 \sim 3.83$ $(\mathrm{m}, 1 \mathrm{H}), 3.01 \sim 2.93(\mathrm{~m}, 2 \mathrm{H}) ; \mathrm{MS}$ (ESI) calcd for $\mathrm{C}_{14} \mathrm{H}_{15} \mathrm{O}_{2} \mathrm{~S}[\mathrm{M}+\mathrm{H}]^{+}$247.1, found 246.9.

Pyridin-2-ylmethyl 4-methylbenzenesulfinate (3ab): colorless oil, $82 \%$ yield. ${ }^{1} \mathrm{H}$ NMR (400 $\left.\mathrm{MHz}, \mathrm{CDCl}_{3}\right) \delta$ : $8.53(\mathrm{~d}, J=6.66 \mathrm{~Hz}, 1 \mathrm{H}), 7.70 \sim 7.65(\mathrm{~m}, 3 \mathrm{H}), 7.39 \sim 7.33$ $(\mathrm{m}, 3 \mathrm{H}), 7.21 \sim 7.18(\mathrm{~m}, 1 \mathrm{H}), 5.16(\mathrm{~d}, J=12.72 \mathrm{~Hz}, 1 \mathrm{H})$, $4.67(\mathrm{~d}, J=12.72 \mathrm{~Hz}, 1 \mathrm{H}), 2.42(\mathrm{~s}, 3 \mathrm{H}) ;{ }^{13} \mathrm{C}$ NMR $(100$ $\left.\mathrm{MHz}, \mathrm{CDCl}_{3}\right) \delta: 156.0,149.3,143.2,141.5,136.9,129.9$, $125.5,123.0,122.3,66.0,21.6$; IR (ATR) $v: 2931,2862$, 1470, 1134, 896, 857, $754 \mathrm{~cm}^{-1}$; HRMS (ESI) calcd for $\mathrm{C}_{13} \mathrm{H}_{14} \mathrm{NO}_{2} \mathrm{~S}[\mathrm{M}+\mathrm{H}]^{+}$248.0740, found 248.0745.

$(1 R, 2 S, 5 R)-2-$ Isopropyl-5-methylcyclohexyl benzenesulfinate $(\mathbf{3 a d}){ }^{[7 \mathrm{bb}]}$ colorless oil, $74 \%$ yield $(d r=1: 1) .{ }^{1} \mathrm{H}$ NMR $\left(600 \mathrm{MHz}, \mathrm{CDCl}_{3}\right) \delta: 7.75 \sim 7.73(\mathrm{~m}, 2 \mathrm{H}), 7.55 \sim$ $7.53(\mathrm{~m}, 3 \mathrm{H}), 4.26 \sim 4.21(\mathrm{~m}, 0.5 \mathrm{H}), 4.18 \sim 4.14(\mathrm{~m}, 0.5 \mathrm{H})$, $2.33 \sim 2.29(\mathrm{~m}, 0.5 \mathrm{H}), 2.18 \sim 2.09(\mathrm{~m}, 1.5 \mathrm{H}), 1.74 \sim 1.67$ $(\mathrm{m}, 2.5 \mathrm{H}), 1.53 \sim 1.45(\mathrm{~m}, 1 \mathrm{H}), 1.42 \sim 1.36(\mathrm{~m}, 1 \mathrm{H}), 1.30 \sim$ 
$1.23(\mathrm{~m}, 1.5 \mathrm{H}), 1.11 \sim 1.02(\mathrm{~m}, 1 \mathrm{H}), 0.99(\mathrm{~d}, J=6.54 \mathrm{~Hz}$, $1.5 \mathrm{H}), 0.93 \sim 0.87(\mathrm{~m}, 6 \mathrm{H}), 0.74(\mathrm{~d}, J=6.90 \mathrm{~Hz}, 1.5 \mathrm{H})$; IR (ATR) $v: 2931,2862,1470,1134,896,857 \mathrm{~cm}^{-1}$; MS (ESI) calcd for $\mathrm{C}_{16} \mathrm{H}_{25} \mathrm{O}_{2} \mathrm{~S}[\mathrm{M}+\mathrm{H}]^{+}$281.2, found 281.0.

$(1 S, 2 R, 4 S)-1,7,7-T r i m e t h y l b i c y c l o[2.2 .1]$ heptan-2-yl benzenesulfinate (3ae): colorless oil, $56 \%$ yield $(d r=1$ : 1). ${ }^{1} \mathrm{H}$ NMR $\left(600 \mathrm{MHz}, \mathrm{CDCl}_{3}\right) \delta: 7.75 \sim 7.71(\mathrm{~m}, 2 \mathrm{H})$, $7.55 \sim 7.53(\mathrm{~m}, 3 \mathrm{H}), 4.62 \sim 4.59(\mathrm{~m}, 0.5 \mathrm{H}), 4.48 \sim 4.46(\mathrm{~m}$, $0.5 \mathrm{H}), 2.40 \sim 2.35(\mathrm{~m}, 0.5 \mathrm{H}), 1.96 \sim 1.89(\mathrm{~m}, 1.5 \mathrm{H})$, $1.78 \sim 1.71(\mathrm{~m}, 1 \mathrm{H}), 1.61 \sim 1.59(\mathrm{~m}, 0.5 \mathrm{H}), 1.34 \sim 1.23(\mathrm{~m}$, $3 \mathrm{H}), 1.08 \sim 1.05(\mathrm{~m}, 0.5 \mathrm{H}), 0.94(\mathrm{~s}, 1.5 \mathrm{H}), 0.87 \sim 0.83(\mathrm{~m}$, $6 \mathrm{H}), 0.75(\mathrm{~s}, 1.5 \mathrm{H}) ;{ }^{13} \mathrm{C} \mathrm{NMR}\left(150 \mathrm{MHz}, \mathrm{CDCl}_{3}\right) \delta: 145.8$, $145.7,131.9,131.8,129.0,128.9,125.3,125.1,84.7,83.7$, $49.8,49.6,47.9,47.8,45.0,44.9,37.6,37.0,28.1,27.9$, 26.8, 26.7, 19.8, 19.7, 18.8, 18.7, 13.4, 13.0; IR (ATR) $v$ : 2985, 1473, 1165, $860 \mathrm{~cm}^{-1}$; HRMS (ESI) calcd for $\mathrm{C}_{16} \mathrm{H}_{22} \mathrm{NaO}_{2} \mathrm{~S}[\mathrm{M}+\mathrm{Na}]^{+}$301.1233, found 301.1234.

\subsection{Large-scale synthesis}

Into a $250 \mathrm{~mL}$ flask disulfide $\mathbf{1 a}$ (4 mmol), alcohol 2a (20 $\mathrm{mL})$, Selectfluor (4 equiv. ) and acetonitrile $(80 \mathrm{~mL})$ were added. The mixture was stirred under air at room temperature for $4 \mathrm{~h}$. Then, the reaction was diluted with $\mathrm{H}_{2} \mathrm{O}(150$ $\mathrm{mL})$ and extracted with EtOAc $(150 \mathrm{~mL} \times 3)$. The combined organic layers were dried with anhydrous $\mathrm{Na}_{2} \mathrm{SO}_{4}$, filtered and concentrated in vacuo. The residue was purified by column chromatography using hexane/EtOAc $(V: V=$ $10: 1)$ as eluent to afford the desired product $\mathbf{3 a}$.

Supporting Information ${ }^{1} \mathrm{H}$ NMR, ${ }^{13} \mathrm{C} \mathrm{NMR}$ and ${ }^{19} \mathrm{~F}$ NMR spectra of compounds 3. The Supporting Information is available free of charge via the Internet at http://siocjournal.cn.

\section{References}

[1] (a) Nguyen, N. L. T.; Vo, H. T.; Duus, F.; Luu, T. X. T. Molecules 2017, 22, 1458 .

(b) Tata, R. R.; Hampton, C. S.; Harmata, M. Adv. Synth. Catal. 2017, 359, 1232.

(c) Yuste, F.; Linares, A. H.; Mastranzo, V. M.; Ortiz, B.; SanchezObregon, R.; Fraile, A.; Ruano, J. L. J. Org. Chem. 2011, 76, 4635. (d) Lujan-Montelongo, J. A.; Estevez, A. O.; Fleming, F. F. Eur. J. Org. Chem. 2015, 1602.

(e) Cao, Z.; Zhu, Q.; Lin, Y. W.; He, W. M. Chin. Chem. Lett. 2019, 30, 2132.

(f) Xu, X.; Chen, D.; Wang, Z. Chin. J. Org. Chem. 2019, 39, 3338 (in Chinese).

(徐金明，陈德茂，王祖利，有机化学, 2019, 39, 3338.)

(g) Li, G.; Yan, Q.; Gan, Z.; Li, Q.; Dou, X.; Yang, D. Org. Lett. 2019, 21, 7938

(h) Gan, Z.; Yan, Q.; Li, G.; Li, Q.; Dou, X.; Li, G. Y.; Yang, D. Adv. Synth. Catal. 2019, 361, 4558.

(i) Gong, X.; Li, G.; Gan, Z.; Yan, Q.; Dou, X.; Yang, D. Asian J. Org. Chem. 2019, 8, 1472 .

(j) Li, G.; Zhang, G.; Deng, X.; Qu, K.; Wang, H.; Wei, W.; Yang, D. Org. Biomol. Chem. 2018, 16, 8015.

(k) Liu, X.; Cui, H.; Yang, D.; Dai, S.; Zhang, T.; Sun, J.; Wei, W.; Wang, H. RSC Adv. 2016, 6, 51830.

[2] (a) Allison, W. S. Acc. Chem. Res. 1976, 9, 293.

(b) Kim, J. H.; Lee, J. O.; Lee, S. K.; Moon, J. W.; You, G. Y.; Kim,

S. J.; Park, S.-H.; Park, J. M.; Lim, S. Y.; Suh, P.-G.; Uhm, K.-O.;
Song, M. S.; Kim, H. S. J. Biol. Chem. 2011, 286, 7567.

(c) Jönsson, T. J.; Murray, M. S.; Johnson, L. C.; Lowther, W. T. J. Biol. Chem. 2008, 283, 23846.

[3] (a) Douglass, I. B. J. Org. Chem. 1965, 30, 633.

(b) Douglass, I. B.; Ward, F. J.; Norton, R. V. J. Org. Chem. 1967, 32,324

(c) Hajipour, A. R.; Falahati, A. R.; Ruoho, A. E. Tetrahedron Lett. 2006, 47, 2717.

(d) Fernandez, I.; Khiar, N.; Roca, A.; Benabra, A.; Alcudia, A.; Espartero, J. L.; Alcudia, F. Tetrahedron Lett. 1999, 40, 2029

[4] Toshikazu, T.; Shigeru, O. Bull. Chem. Soc. Jpn. 1982, 55, 3937.

[5] D’Oca, M. G. M.; Russowsky, D.; Canto, K.; Gressler, T.; Goncüalves, R. S. Org. Lett. 2002, 4, 1763.

[6] Tranquilino, A.; Andrade, S. R. C. P.; da Silva, A. P. M.; Menezes, P. H.; Oliveira, R. A. Tetrahedron Lett. 2017, 58, 1265.

[7] (a) Field, L.; Hoelzel, C. B.; Locke, J. M. J. Am. Chem. Soc. 1962 , 84,847 .

(b) Brownbridge, P.; Jowett, I. C. Synthesis 1988, 252

(c) Xia, M.; Chen, Z. C. Synth. Commun. 1997, 27, 1301

(d) Zhou, C.; Tan, Z.; Jiang, H.; Zhang, M. Green Chem. 2018, 20, 1992.

(e) Douglass, I. B. J. Org. Chem. 1973, 39, 563.

(f) Brownbridge, P.; Jowett, I. C. Synthesis 1988, 252.

(g) Wei, J.; Sun, Z. Org. Lett. 2015, 17, 5396.

[8] (a) Du, B.; Li, Z.; Qian, P.; Han, J. L.; Pan, Y. Chem.-Asian J. 2016, 11,478 .

(b) Du, B.; Wang, W.; Wang, Y.; Qi, Z.; Tian, J.; Zhou, J.; Wang, X.; Han, J. L.; Ma, J.; Pan, Y. Chem.-Asian J. 2018, 13, 404.

[9] (a) Ai, C.; Shen, H.; Song, D.; Li, Y.; Yi, X.; Wang, Z.; Ling, F.; Zhong, W. Green Chem. 2019, 21, 5528.

(b) Gong, F.; Ju, F.; Zuo, L.; Wang, Q.; Li, R.; Hu, J.; Li, Z.; Takfaoui, A.; Lei, A. J. Chin. Chem. Soc. 2020, 67, 192.

[10] (a) He, Y.; Zhang, J.; Xu, L.; Wei, Y. Tetrahedron Lett. 2020, 61, 151631

(b) Lavey, C. F.; Hesk, D.; Hendershot, S.; Koharski, D.; Saluja, S.; Namara, P. M. J. Labelled Compd. Radiopharm. 2007, 50, 264.

(c) Ji, Y.; Wang, M.; Li, H.; Liu, Y.; Wu, Y. Eur. J. Org. Chem. 2016, 4077.

(d) Dahn, H.; Toan, V. V.; Ung-Truong, M. Magn. Reson. Chem. 1991, 29, 897.

[11] Nyffeler, P. T.; Durón, S. G.; Burkart, M. D.; Vincent, S. P.; Wong, C. H. Angew. Chem., Int. Ed. 2005, 44, 192.

[12] (a) Cheng, H. G.; Yin, G. Chem 2019, 5, 1022.

(b) Szpera, R.; Moseley, D. F. J.; Smith, L. B.; Sterling, A. J.; Gouverneur, V. Angew. Chem., Int. Ed. 2019, 58, 14824.

(c) Wood, S. H.; Etridge, S.; Kennedy, A. R.; Percy, J. M.; Nelson, D. J. Chem.-Eur. J. 2019, 25, 5574.

(d) Xu, J.; Kuang, Z.; Song, Q. Chin. Chem. Lett. 2018, 29, 963.

(e) Shibata, N.; Suzuki, E.; Takeuchi, Y. J. Am. Chem. Soc. 2000 , 122, 10728.

(f) Mei, H.; Remete, A. M.; Zou, Y.; Moriwaki, H.; Fustero, S.; Kiss, L.; Soloshonok, V. A.; Han, J. Chin. Chem. Lett. 2020, DOI: org/10.1016/j.cclet.2020.03.050.

[13] (a) Niu, L.; Liu, J.; Liang, X. A.; Wang, S.; Lei, A. Nat. Commun. 2019, 10, 467.

(b) Liang, X. A.; Niu, L.; Wang, S.; Liu, J.; Lei, A. Org. Lett. 2019, 21, 2441.

(c) Xie, L. Y.; Qiu, J.; Peng, S.; Liu, K. J.; Wang, Z.; Ding, M. H.; Wang, Y.; Cao, Z.; He, W. M. Green Chem. 2018, 20, 760.

(d) Zhou, J.; Zou, Y.; Zhou, P.; Chen, Z.; Li, J. Org. Chem. Front. 2019, 6, 1594.

(e) Xie, L. Y.; Peng, S.; Liu, F.; Yi, J. Y.; Wang, M.; Tang, Z.; Xu, X.; He, W. M. Adv. Synth. Catal. 2018, 360, 4259.

(f) Galloway, J. D.; Mai, D. N.; Baxter, R. D. Org. Lett. 2017, 19 , 5772 .

(g) Wang, C. X.; Cai, J. W.; Zhang, M.; Zhao, X. M. J. Org. Chem. 2017, 82, 1260.

(h) Zhou, G.; Tian, Y. W.; Zhao, X. M.; Dan, W. Y. Org. Lett. 2018, 
20, 4858.

(i) Hu, J.; Zhou, G.; Tian, Y.; Zhao, X. Org. Biomol. Chem. 2019, 17, 6342 .

(j) Yuan, J. W.; Zhu, J. L.; Li, B.; Yang, L. Y.; Mao, P.; Zhang, S. R.; Li, Y. C.; Qu, L. B. Org. Biomol. Chem. 2019, 17, 10178

(k) Yuan, J.; Zeng, F.; Mai, W.; Yang, L.; Xiao, Y.; Mao, P.; Wei, D. Org. Biomol. Chem. 2019, 17, 5038

(1) Mai, W. P.; Yuan, J. W.; Zhu, J. L.; Li, Q. Q.; Yang, L. R.; Xiao, Y. M.; Mao, P.; Qu, L. B. ChemistrySelect 2019, 4, 11066.

(m) Mei, H. B.; Liu, J.; Pajkert, R.; Röschenthaler, G. V.; Han, J. L.
Org. Biomol. Chem. 2020, 18, 3761.

[14] Yadav, J. S.; Reddy, B. V. S.; Reddy, Y. J. Tetrahedron Lett. 2007, 48, 7034.

[15] Kirihara, M.; Naito, S.; Ishizuka, Y.; Hanai, H.; Noguchi, T. Tetrahedron Lett. 2011, 52, 3086.

[16] Vincent, S. P.; Burkart, M. D.; Tsai, C. Y.; Zhang, Z.; Wong, C. H. J. Org. Chem. 1999, 64, 5264.

[17] Tota, A.; John-Campbell, S. S.; Briggs, E. L.; Estévez, G. O.; Afonso, M.; Degennaro, L.; Luisi, R.; Bull, J. A. Org. Lett. 2018, 20, 2599.

(Zhao, C.) 